\title{
Correlation Functions Formed by a Femtosecond Pulse Interferometer
}

\author{
M. Cui ${ }^{a}$, N. Bhattacharya ${ }^{a}$, H.P. $\operatorname{Urbach}^{a}$, and S.A. van den $\operatorname{Berg}^{b}$ \\ ${ }^{a}$ Optics Research Group, Department of Applied Sciences, Technical University Delft, \\ Lorentzweg 1, 2628 CJ Delft, The Netherlands \\ ${ }^{b}$ NMi Van Swinden Laboratory, Thijsseweg 11, 2629 JA Delft, The Netherlands
}

\begin{abstract}
We experimentally demonstrate that a stabilized femtosecond frequency comb can be applied as a tool for distance measurement. The scheme is based on optical interference between individual pulses in a Michelson type interferometer. The cross-correlation functions between individual pulses with a distance of around 15 meter and 30 meter are observed and analysed.
\end{abstract}

\section{INTRODUCTION}

Since the invention of the laser numerous applications of lasers in the field of distance measurement have been developed. Using traditional techniques such as classical interferometry, accuracy at the nanometer level can be reached. To measure absolute length, the value of the measured length has to be known beforehand with sub-wavelength accuracy, because of the ambiguity of the interferometric measurement results. In 2004, a novel scheme for measuring long distances in vacuum with a stabilized femtosecond frequency comb was proposed by Ye. ${ }^{1}$ The scheme is based on optical interference between individual pulses in a Michelson type interferometer. Interference is possible because the carrier-envelope stabilized pulses have a fixed phase relation with respect to each other. In this paper we present our current experiments using this principle. We have measured the cross-correlation between pulses with path length differences of 15 and 30 meters. The accuracy, limitations and possible improvements to this experiment will be discussed.

\section{MEASUREMENT PRINCIPLE}

A frequency comb is generated from a phase-stabilized mode-locked femtosecond laser. ${ }^{\text {? }}$ The periodic train of pulses emitted from the laser has its counterpart in the frequency domain as a comb of equidistant modes with a mutual separation equal to the repetition frequency $f_{r}$. The difference between the group velocity and phase velocity gives rise to a pulse to pulse phase shift $\Delta \phi$ between the carrier wave and the envelope. This phase shift results in an offset frequency $f_{0}$, generally referred to as the carrier-envelope offset (CEO) frequency. This is illustrated in Fig. 1. The CEO frequency and the repetition frequency are related by $f_{0}=(\Delta \phi / 2 \pi) f_{r}$. When both $f_{r}$ and $f_{0}$ are stabilized to a reference value, the distance between the two adjacent pulses $l_{p p}$ is fixed and the carrier-envelope offset is stabilized.

For distance measurement the pulse train is sent into a Michelson interferometer consisting of a measurement arm and a reference arm. A coherence maximum in the interferometer output is obtained once the path length difference between the arms is a multiple of $l_{p p}$. This can be accomplished by applying a calibrated delay line to tune the path length difference to a multiple of the interpulse distance. If the range of the delay line exceeds $l_{p p} / 2$, spatial overlap between the pulses can always be accomplished. The non-ambiguity range of the distance measurement is determined by $l_{p p}$ and is of the order of $1 \mathrm{~m}$ for most fs laser sources. The required initial value of the distance to be measured, can be easily determined, e.g. with a time-of-flight measurement. This scheme may be viewed as an extension of multi-wavelength interferometry, with the phase-stabilized mode-locked laser as a multi-wavelength source.

In a previous experiment ${ }^{2}$ we have experimentally demonstrated that an optical frequency comb laser can be applied for interferometric measurement of displacement. For a short displacement of $15 \mathrm{~cm}$ the results agree within less than half a wavelength with an independent measurement performed with a calibrated He-Ne laser

Advanced Optical and Mechanical Technologies in Telescopes and Instrumentation, edited by Eli Atad-Ettedgui, Dietrich Lemke, Proc. of SPIE Vol. 7018, 70183Q, (2008) · 0277-786X/08/\$18 · doi: 10.1117/12.789097 

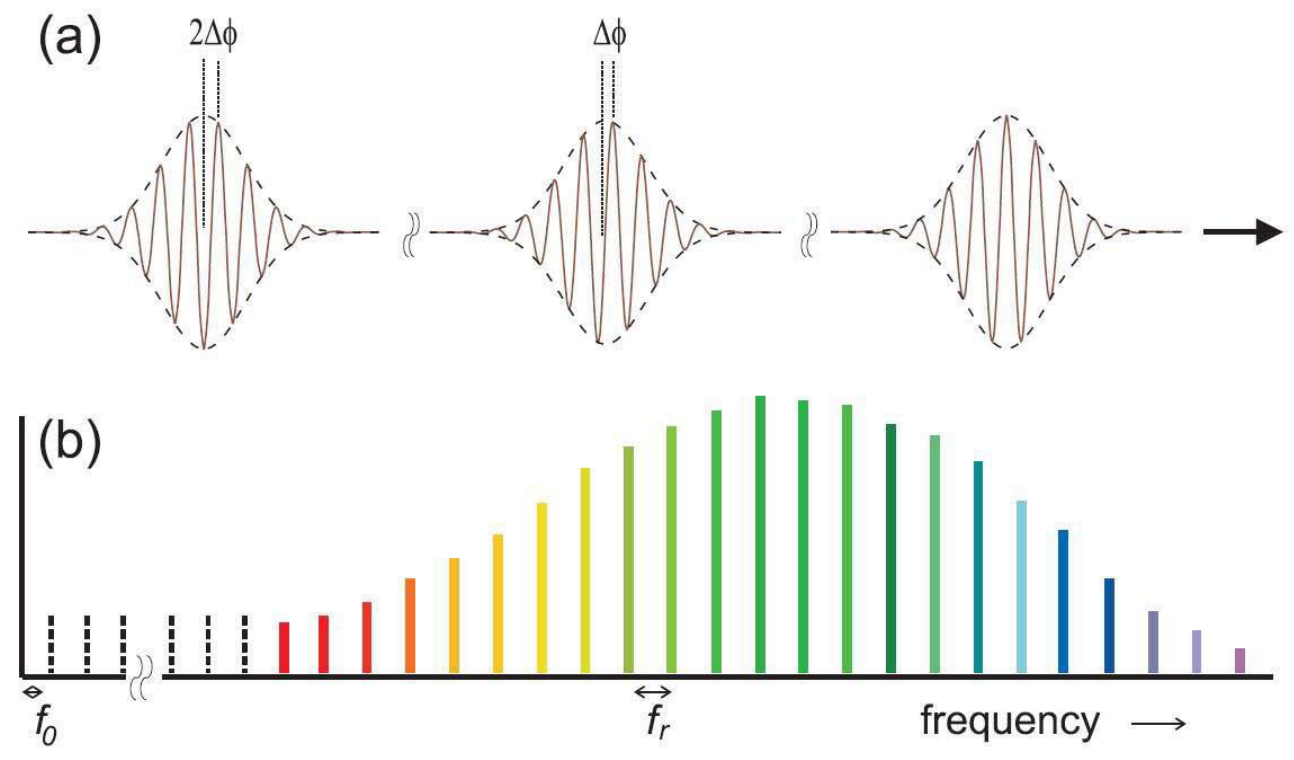

Figure 1. a) Illustration of the carrier-envelope phase shift in the time domain. A pulse to pulse phase shift $\Delta \phi$ is observed. b) Corresponding optical frequency spectrum with $f_{0}$ the offset frequency due to the carrier-envelope phase shift, and $f_{r}$ the pulse repetition rate

interferometer. The present measurement aims to increase the measurement distance to a much longer range (tens of meters).

\section{EXPERIMENTAL}

A schematic of the actual experimental setup is shown in Fig. 2 The experiment is performed in two adjacent rooms. In the room on the left, a mode-locked Ti:Sapphire laser generates ultrashort pulses with durations about $40 \mathrm{fs}$. The output wavelength of the pulse is centered at $820 \mathrm{~nm}$ with a bandwidth of $20 \mathrm{~nm}$. The repetition frequency is approximately $1 \mathrm{GHz}$, corresponding to a cavity length of the laser of about $15 \mathrm{~cm}$. The offset frequency is fixed at $f_{0}=180 \mathrm{MHz}$. Both the repetition frequency and the carrier-envelope-offset frequency are locked and referenced to a cesium clock. Two cirved mirrors are used to minimize the divergence of the beam. The ultrashort laser beam passes through a hole in the wall and is sent along the two arms of the interferometer by the beam splitter in the room on the right, the measurement room. One part of the pulse goes into a short reference arm with length $l_{r}$. The length of this arm can be varied with a translation stage of $15 \mathrm{~cm}$ to obtain a spatial overlap of the pulses. The cross-correlation signal is acquired by scanning with a piezo-element. The other part of the pulse is reflected by two mirrors and travels to a long measurement arm. The retroreflector is fixed on a cart moving along a $50 \mathrm{~m}$ measurement bench. The beams reflected from both arms are made to coincide with each other and are focused onto an avalanche photodiode (APD). When the reference arm is set at the distance

$$
l_{m}=l_{r}+n \cdot l_{p p} / 2
$$

where $n$ is an integer number, a cross-correlation function is measured.

\section{RESULTS AND DISCUSSION}

The interferometer was aligned such that the retroreflector on the $50 \mathrm{~m}$ measurement bench was at the single-path distances of $0.9 \mathrm{~m}, 15.9 \mathrm{~m}$ and $30.9 \mathrm{~m}$ and correlation patterns were recorded. This corresponds to correlation between the two pulses with $n=6, n=106$ and $n=206$ in Eqn.1. The short distance of $0.9 \mathrm{~m}$ is inherent in the setup due to the beam transport into the long arm. Fig.3 shows the recorded cross-correlation patterns at the three distances. The theoretical maximum contrast of the correlation function should be 0.5 . We can see that, 


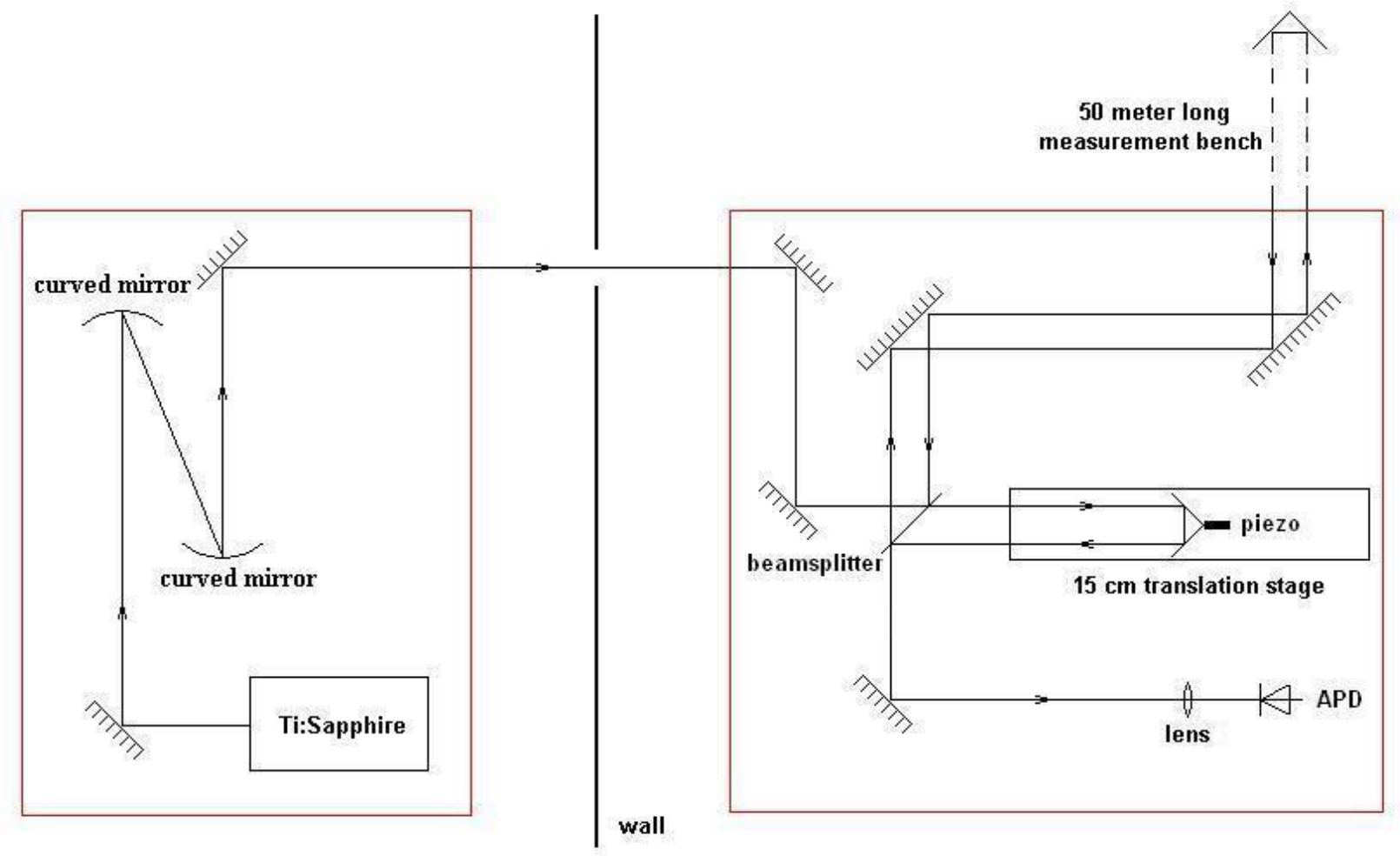

Figure 2. Schematic of the experimental setup. Ultrashort laser beam is sent into an interferometer. One part of the pulse goes into a short reference arm with length $l_{r}$. The other part of the pulse travels to a $50 \mathrm{~m}$ measurement bench. The cross-correlation signal is acquired by scanning with a piezo-element in the reference arm. The beams reflected from both arms are made to coincide with each other and are focused onto an avalanche photodiode (APD) thus cross-correlation functions can be measured.

the contrast of the correlation function at $n=6$ is 0.38 , where the reduction may come from the misalignment and the unequal intensity from both arms. At a distance of $n=106$, the contrast reduces to around 0.2 , but still clear enough to fit a Gaussian and find the maximum. As the measurement arm increases to $30.9 \mathrm{~m}$, still the correlation function can be observed, but the contrast is as low as only 0.09. It is clearly seen that after propagating around $60 \mathrm{~m}$ (back and forth) in air the pulse is significently broadened. This is due to the group delay dispersion of air.

In the next step, we plan to use two He-Ne lasers interferometers to give independent measurements to the reference arm and the measurement arm. The reference arm being at the maximum only $15 \mathrm{~cm}$ long, corresponding to the cavity length of the laser in air, can be measured with an high accuracy. The long measurement arm can be checked by another He-Ne laser which has a lower accuracy but a longer range.

We find that several aspects of the experiment need attention so that the accuracy and range of the measurements can be improved. One of them is beam shape distortion. The wave-front of the pulse is distorted during the propagation through air and optical components hence influences the contrast of the correlation. Although two cirved mirrors are used for beam shaping, the divergence in the long arm of the interferometer is still significant and there is room for improvement. Another aspect of concern is that the pulse is both chirped and broadened during propagation, and the spatial dispersions such as spatial chirp, wavefront tilt and angular dispersion from optical elements seriously influence the pulse shape and thus the cross-correlation patterns. The experimental rooms are temperature controlled and the pressure variations are monitored. However, the refractive index of air is an important and limiting parameter for this kind of measurement, and strongly depend on 


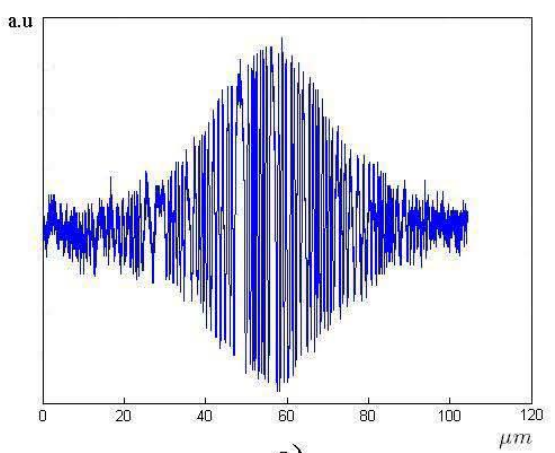

a)

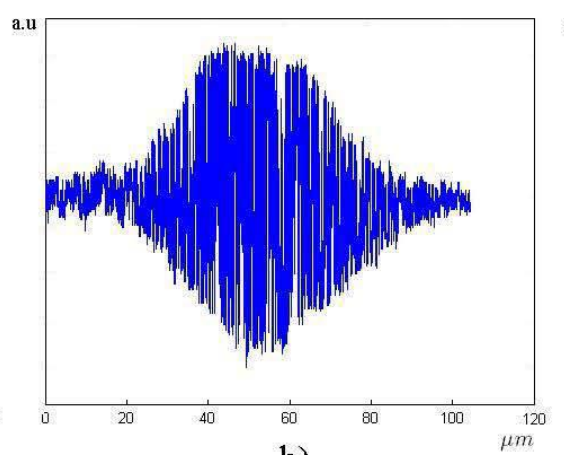

b)

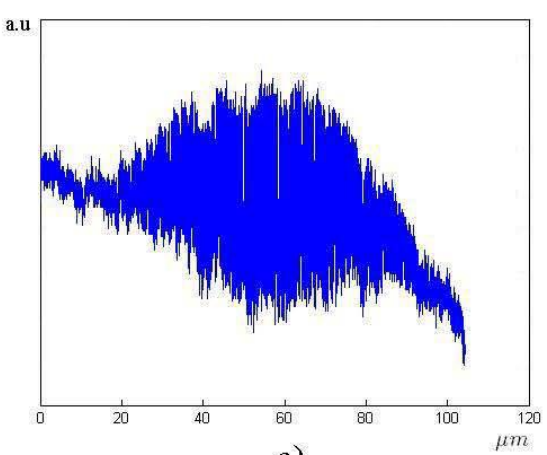

c)

Figure 3. The cross-correlation patterns at a) $n=6$, b) $n=106$ and c) $n=206$ corresponding to distances between the two arms around a) $0.9 \mathrm{~m}$, b) $15.9 \mathrm{~m}$ and c) $30.9 \mathrm{~m}$ respectively. The contrast of the cross-correlation function decreases as the displacement between the two arms getting larger.

the environmental conditions. We need to keep in mind that $1^{\circ} \mathrm{C}$ change in temperature will cause a $14.6 \mu m$ error in a measurement of 15 meters, and $1 \mathrm{hPa}$ fluactuation on air pressure courses an error of $4 \mu \mathrm{m}$. This was not an issue for the initial proposal of the experiment which was intended for distance measurements between satellites, but makes demonstratating the principle in a laboratory a challenge.

In conclusion, we have experimentally demonstrated that cross-correlation functions can be observed for a distance of tens of meters between the two arms in an ultrashort pulse interferometer. This method can be used as a promising tool for long distance measurements. We plan to extend the range and accuracy with further improvements to the setup.

\section{REFERENCES}

1. J. Ye, "Absolute measurement of a long, arbitrary distance to less than an optical fringe", Opt. Lett. 29, 1153 (2004).

2. D.Jones, S.A.Diddams, J.K.Ranka, A.Stentz, R.S.Windeler, J.L.Hall and S.T.Cundiff, "Carrier-envelope phase control of femtosecond mode-locked lasers and direct optical frequency sysnthesis", Science 288, 635 (2000).

3. M. Cui, R. N. Schouten, N. Bhattacharya and S. A. van den Berg, "Experimental demonstration of distance measurement with a femtosecond frequency comb laser", J. Europ. Opt. Soc. Rap. Public. 1, 08003 (2008). 